\title{
Interessenpolitik und Werteorientierung - Perspektiven der Gewerkschaftsarbeit
}

\author{
Hans-Jürgen Arlt \\ Wolfgang Schroeder \\ Klaus-W. West
}

\section{AM ANFANG STEHT GLAUB- WÜRDIGKEIT}

Betriebsräte, Personalräte und Gewerkschaften vertreten Interessen. In unserer Gesellschaft haben Interessen einen zwiespältigen Status. Einerseits genießen sie einen guten Ruf. Dass jeder seine Interessen artikulieren und zur Geltung bringen darf, war historisch der große Fortschritt, den die moderne bürgerliche Gesellschaft für sich reklamierte. Diese positive Bewertung gipfelte in der Aussage, das allgemeine Beste verwirkliche sich gerade dadurch, dass jede und jeder individuelle Interessen verfolge. Andererseits sehen sich Interessen, besonders wenn sie organisiert auftreten, massiver Kritik ausgesetzt: Der Staat als Repräsentant des Allgemeinen werde von Interessen belagert, in seiner Entscheidungsund Handlungsfähigkeit eingeschränkt.

Weil Politik auf die allgemeinen Angelegenheiten, auf das kollektiv Verbindliche zielt, müssen sich Interessen in der politischen Kommunikation an der Frage messen lassen, ob sie dem Allgemeinwohl förderlich sind - wobei das Allgemeinwohl selbst keine eindeutige, sondern nur eine kontrovers bestimmbare Bezugsgröße darstellt. Eine zuverlässigere Verbindung zwischen Einzelinteressen und mehrheitlich anerkannten Vorzugspositionen kann über Werte gelingen. Werte sind gesellschaftlich anerkannte Präferenzen. Sie können im Widerstreit zueinander stehen, aber erst einmal bestreitet niemand, dass Freiheit der Unfreiheit, Gerechtigkeit der Ungerechtigkeit, Sicherheit der Unsicherheit vorzuziehen ist.

In der politischen Öffentlichkeit finden solche Interessen leichter Gehör, die sich darauf berufen, ihre Missachtung bedeute, Werte zu verletzen. So steht beispielsweise die Kritik, dass die soziale Gerechtigkeit unter die Räder gerate, im Mittelpunkt vieler gewerkschaftlicher Auftritte. Für Interessenorganisationen ist die Anschlussfähigkeit an Werte ein wichtiges Moment ihrer Außendarstellung. Vor allem aber verlieren Interessenorganisationen an innerer Bindungskraft, wenn sie über die vertretenen Interessen hinaus nicht zugleich gemeinsame Werte ihrer Mitglieder repräsentieren. Problem- und kostenlos lässt sich Interessenpolitik nicht als Wertepolitik profilieren: Sie setzt sich einem permanenten Glaubwürdigkeitstest aus. Die programmatischen Ziele, die sie verfolgt, und die Instrumente, welche sie dabei einsetzt, werden intern und extern daraufhin überprüft, ob sie mit den proklamierten Werten vereinbar sind.

\section{KONTINUITÄT DER WERTE, FLEXIBILITÄT DER ZIELE UND INSTRUMENTE}

Wenn die Ziele gewerkschaftlicher Politik veränderten Realitäten nicht Rechnung tragen, schwächt dies die Überzeugungskraft der Gewerkschaften und eröffnet konservativer oder wirtschaftsliberaler Politik die Möglichkeit, Anliegen der Gewerkschaften zu besetzen oder Forderungen zu entkräften. Es ist beispielsweise nicht gut, wenn betriebsbezogene Abschlüsse mit den Betriebsräten in Unternehmen durchgesetzt werden, Gewerkschaften sich dazu in der Öffentlichkeit jedoch nicht bekennen. Jedenfalls dürfen die Vorstellungen für die Ausgestaltung der Tarifpolitik oder der sozialen Sicherheit nicht in anhaltenden Wi- derspruch geraten mit den Erfahrungen und Erwartungen der Mitglieder. Differenzen im Einzelnen sind unvermeidlich. Konkrete politische Entscheidungen werden stets in einem Korridor von Zielkonflikten zu treffen sein, die nicht in die Harmonie allseitiger Interessenbefriedigung auflösbar sind. Aber ein politisches Controlling, ob die Entscheidungen an die vorherrschenden Erfahrungen und Erwartungen der Mitglieder - und übrigens auch potenzieller Mitglieder - anschlussfähig und vermittelbar sind, muss stets mitlaufen. Mit einer vormundschaftlichen Position der höheren Wahrheit lässt sich nämlich nur die eigene politische Biografie vor gefühlten Verlusten schützen, aber kein neues Mitglied gewinnen.

Die Megatrends der Gegenwart, Individualisierung, Digitalisierung und Globalisierung, lassen keinen Gesellschaftsbereich unberührt. Wirtschaftliche, soziale, kulturelle und politische Veränderungsprozesse rufen neue Bedrohungen und neue Chancen hervor. Unsere Grundthese lautet: Für die gewerkschaftliche Arbeit gilt eine Kontinuität der Werte Freiheit, Gerechtigkeit und Solidarität. Aber sowohl deren jeweiliger Bedeutungsgehalt als auch ihr Verhält-

\footnotetext{
Hans-Jürgen Arlt, Dr., Pressesprecher DGB Hamburg, Publizist in Berlin. e-mail: H-J.Arlt@gmx.de Wolfgang Schroeder, Dr., Professor für Politikwissenschaft, Universität Kassel. e-mail: Wolfgang.schroeder@uni-kassel.de Klaus-W. West, Dr., Büroleiter des Vorsitzenden der IG BCE in Hannover. e-mail: klaus.west@igbce.de
} 
nis zu den politischen Zielen und Instrumenten sind immer wieder aktuell $\mathrm{zu}$ klären und zu erklären. Dabei können die programmatischen Ziele und die Instrumente gewerkschaftlicher Politik in einer sich rapide wandelnden Umwelt nicht identisch bleiben: „Nur wer sich ändert, bleibt sich treu“ (Wolf Biermann).

\section{PRODUZENTEN DER POLITIK- VERDROSSENHEIT}

Es wäre eine einseitige und unfaire Bewertung, würden nur die Schwierigkeiten der Arbeitnehmerorganisationen thematisiert, Konsistenz und Glaubwürdigkeit in ihrem politischen Handeln zu erhalten bzw. wiederzugewinnen. Parteien und Arbeitgeber haben vergleichbare, häufig sogar noch größere Probleme, ihre Interessenpolitik als „wertvoll“ zu kommunizieren und zu praktizieren. Arbeitgeber, von ihrer Funktion her zunächst ohnehin auf nichts anderes als den Mehr-Wert fixiert, betonen traditionell den Wert der Freiheit - aber nur in der negativen, auf die „Freiheit der Marktkräfte" zugeschnittenen Form. Rücksichten auf Arbeit und Umwelt müssen allzu oft gegen eine Shareholder-Fixierung durchgesetzt werden, die Menschen und Natur zu bloßen Instrumenten der Gewinnmaximierung degradiert.

In der Politik der Parteien werden Werte gewöhnlich den hochgradig spezialisierten Bereichspolitiken in Form eines weltanschaulichen Vorworts vorgeschaltet. Die Werteperspektive durchdringt und organisiert die komplizierten Bereichsfragen nicht. Die Sinnfälligkeit der Werte ist für die Bereichspolitiken nicht erkennbar. Dafür ist die Gesundheitsreform im Jahr 2006 ein gutes Beispiel. Reformtechnische Details und Verfahrensfragen standen im Zentrum. So erscheinen Werte und Fachpolitiken als getrennt voneinander. Die Generalrichtung ist nicht mehr klar.

Auch eine Kommunikationsstrategie, wie sie Bundeskanzlerin Angela Merkel in ihrer Regierungserklärung versucht hat über Werte zu reden, um über Interessen schweigen zu können -, geht nicht auf. „Mehr Freiheit", „neue Gerechtigkeit“, „Verlässlichkeit als Markenzeichen“, „mehr Ehrlichkeit" waren Merkels Fixsterne am Wertehimmel, die ihre Regierungskunst über die dunklen Interessen-Wolken hinausheben sollten. Sie verlangte sogar ausdrücklich, von Interessen abzusehen: „Überraschen wir uns damit, dass wir die großen Fragen nicht immer aufgegliedert nach Einzelgruppen und Interessen angehen ". ${ }^{1}$ Für wessen Interessen wird das eine böse Überraschung?

Zusammengefasst: Interessenpolitiker, die keinen glaubwürdigen Bezug zu gesellschaftlich anerkannten Werten aufrecht erhalten können, und Werteprediger, welche angesichts widerstreitender Interessen Sand in die Augen zu streuen versuchen, sind an der Produktion von Politikverdrossenheit beteiligt.

\section{ALTE UND NEUE WERTETRIAS, NEGATIVE UND POSITIVE FREIHEIT}

Die alte Wertetrias der Gewerkschaften, Freiheit, soziale Gerechtigkeit und Solidarität, fand in Zielen wie „gute Arbeit“, „gerechte Löhne" und „soziale Sicherheit“ ihre gewerkschaftliche politische Form. Als Instrumente dienten Mitbestimmung, Tarifpolitik und das Engagement für Demokratie und Sozialstaat.

Die alte Wertetrias der Gewerkschaften stand auf den festen Fundamenten der Arbeit in den industriellen und den staatlichen Großbetrieben, einer homogenen Arbeitnehmergesellschaft mit der öffentlichen sowie privaten Arbeitsteilung zwischen Mann und Frau und eines starken Staates in einem nationalen Politikrahmen. Gewerkschaften konnten sich auf den Sozialstaat und den Flächentarifvertrag stützen, um ihrer Vorstellung von Vollbeschäftigung und sozialer Demokratie näher zu kommen.

Die sich wechselseitig bedingenden Werte Freiheit, Gerechtigkeit und Solidarität begründen eine Praxis, die negative wie positive Freiheit umfasst. Historisch begann dies mit der Verteidigung negativer Freiheit im Unternehmen, als Schutz vor unternehmerischer Willkür und Ausbeutung. Es folgte der Kampf für die positive Bestimmung von Freiheit über die freie Zeit jenseits der Arbeit - z. B. der Kampf um die 40 Stunden-Woche - bis zu einem Einkommen, das es erlaubte, die freie Zeit nutzen zu können.

Freiheit realisierte sich durch die Solidarität der abhängig Beschäftigten. Somit war Solidarität, verstanden als gegenseitige Hilfe und als gemeinsam getragene soziale Sicherheit, Bedingung für die Freiheit der Arbeitnehmerinnen und Arbeitnehmer. Soziale Gerechtigkeit, verstanden als sozialgerechte materielle Teilhabe, war die Bedingung für positive Freiheit, d.h. für die
Möglichkeit, aufgrund einer akzeptablen und sicheren materiellen Grundlage ein selbstbestimmtes Leben zu führen. Materielle soziale Gerechtigkeit schließlich war eine wesentliche Bedingung für Solidarität, die Ungleichheit begrenzte und eine Gleichheit anstrebte, die es ermöglichte, sich mit anderen solidarisch zu verhalten. Die Kraft zur Solidarität basierte auf vergleichbaren Einkommen und vergleichbaren Arbeitsbedingungen.

Die alte Trias von Freiheit, Gerechtigkeit und Solidarität ist die neue. Das heißt auch: Die Werte sind im Grunde die gleichen geblieben, ihre Ausdrucksformen haben sich allerdings ausdifferenziert. So lässt sich heute nicht mehr von "der" sozialen Gerechtigkeit sprechen, sondern wir sprechen über Teilhabe-, Bedarfs-, Leistungs-, Verteilungs-, Generationen-, Geschlechter- und Chancengerechtigkeit. Entscheidend ist, dass die Ziele und Instrumente für die Verwirklichung dieser Werte andere geworden sind.

Der sich verändernde Handlungsrahmen für gewerkschaftliche Aktivitäten ist durch den Übergang von der alten Industriegesellschaft auf eine stärker mittel- und kleinbetriebliche Dienstleistungs- und Wissensökonomie mit weiterhin starkem industriellen Kern geprägt. Diese Verschiebung verlangt auch eine neue Ziel- und Instrumentendiskussion. Dabei spielen sich diese Neujustierungsprozesse im Rahmen einer heterogenen Arbeitnehmergesellschaft $\mathrm{ab}$, die durch einen in seinen Handlungsmöglichkeiten eingeschränkten Nationalstaat, einen europäisierten Politikrahmen sowie durch eine veränderte geschlechtliche Arbeitsteilung geprägt ist.

\section{ZEITGEMÄßE GEWERKSCHAFTLICHE WERTEPOLITIK}

Es ist wichtig, die Akzentverschiebungen zwischen einer zeitgemäßen und einer traditionellen Wertepolitik der Gewerkschaften zu verdeutlichen. Letztere lebte aus der Opposition gegen die Freiheitsvorstellungen der Konservativen und Wirtschaftsliberalen und aus der Hervorhebung der sozialen Gerechtigkeit und Solidarität gegenüber der negativen Freiheit und der

\footnotetext{
Merkel, Angela (2005): Regierungserklärung als Bundeskanzlerin, 30.11.05, Presse- und Informationsamt (Hrsg.), Manuskript S. 5.
} 
Freiheit der Wirtschaftssubjekte. Freiheit war gewissermaßen ein weißer Kommunikationsfleck im Diskurs der Gewerkschaften, sie haben diesen Begriff mit Blick auf die Sozial- und Wirtschaftspolitik nicht offensiv kommuniziert.

Heute wird positive Freiheit als gewerkschaftspolitisches Ziel wichtiger. Dies ist ein Erfolg von mehr als 50 Jahren Demokratie, und dies verlangt neue Zielsetzungen ordnungspolitischen Charakters. Die Reformen des deutschen Modells müssen auch die Zielsetzung haben, die Freiheit im Sinne von mehr Teilhabe und Zugängen in Wirtschaft und Gesellschaft zu vergrößern. Gewiss braucht Freiheit eine soziale Grundlage. Neben einem anständigen und angemessenen Einkommen müssen Arbeitnehmerinnen und Arbeitnehmer stärker ihre Fähigkeiten und Kompetenzen in die Arbeit einbringen können. Die Erweiterung der Betätigungs- und Mitsprachemöglichkeiten ist der Prüfstein für praktisch verstandene Freiheit. Dafür sind die Bedingungen besser denn je, denn es ist heute nicht mehr zwingend erforderlich und teilweise sogar kontraproduktiv, Arbeitsabläufe nach tayloristischem Muster zu organisieren. Beteiligungsoffene Unternehmenskulturen sind $\mathrm{zu}$ einer realen Möglichkeit geworden.

Wie kann in Zeiten von abstiegsbedrohten Mittelschichten und prekären Lebenslagen am unteren Rand der sozialen Hierarchie bei einem Mehr an positiver Freiheit Solidarität gesichert werden? Sicherlich können im Interesse der Solidarität die Differenzierungen von positiver Freiheit nicht zurückgenommen werden, die positiven Bestimmungen von Freiheit müssen vielmehr mit Solidarität zusammengeschaltet werden, um selbstbezogene Verhaltensweisen einzugrenzen. So kann die Solidaritätsfähigkeit und -bereitschaft der Mittelklassen erhalten, die Verantwortung der Spitzen der Gesellschaft für das Gemeinwohl eingefordert werden. Es ist aber ebenso wichtig, dass die sozial Benachteiligten und die am meisten von existenziellen Risiken Bedrohten darin aktiv unterstützt werden, aus der Rolle von Opfern heraus zu finden und Kraft zur Eigeninitiative zu gewinnen. Was bedeutet dies? Kurzfristig müssen dafür institutionelle Regelungen geschaffen werden, die die Integration in die Arbeit erleichtern; mittelfristig muss Bildung die Menschen nicht nur fachlich qualifizieren, sondern auch zu mehr Selbstorganisation befähigen. „So- zialhilfekarrieren“" zeigen, dass alleine materielle Hilfen nicht vor „Integrationssackgassen“ schützen. Als Ziel geht es darum, die Weichen zu stellen, dass es wieder auf Leistung basierende Aufstiegsmöglichkeiten gibt.

Zeitgemäße Wertepolitik heißt, neben der Verteilungsgerechtigkeit auf die Teilhabechancen zu setzen, die die Möglichkeit bieten, durch eine gute Ausbildung den Weg zu einem guten Arbeitsplatz zu finden. Die Konservativen heben nach wie vor den Gedanken der sozialen Selektion und die produktive gesellschaftliche Bedeutung von Wettbewerb und sozialer Ungleichheit hervor. Der klassische wertepolitische Ansatz der Gewerkschaften favorisiert die materielle Verteilungsgerechtigkeit, auf die dann kulturelle Teilhabechancen aufgesattelt werden. Dies zeigt sich insbesondere in Fragen der beruflichen Weiterbildung. Aber heute brauchen gerade auch die sozial schlechter Gestellten und die geringer Qualifizierten den Zugang zu mehr Weiterbildungsmöglichkeiten. Bessere Zugänge, mehr Teilhabechancen sind keine Schönwetterforderung, sondern ein Kernelement der Arbeits- und Gesellschaftspolitik.

Es sind zwei Dimensionen, die über die Beteiligung in modernen Gesellschaften entscheiden. Der erste Zugang erfolgt über eine qualitative Dimension der Beteiligung, um sich selbst Anrechte und Einkommenschancen zu erwerben. Dies verläuft über Ausbildung, Qualifikation und Beschäftigung. Der zweite Zugang eröffnet sich über eine quantitative Dimension, die über die Beteiligung im Sinne fairer Einkommensund Vermögensverteilung entscheidet. Beide Zugänge führen zu einer zeitgemäßen Ausgestaltung der sozialen Gerechtigkeit.

Gewerkschaften wissen darum, dass beide Zugänge geschaffen und garantiert werden müssen. Deshalb bedeutet die Differenzierung des Gerechtigkeitsbegriffes nicht die Verdrängung einer Gerechtigkeitsdimension zugunsten einer anderen, sondern sie erfordert einen Prüfprozess, um der jeweiligen Situation gerecht zu werden. Ausschlaggebend sind die Bedürfnisse der Betroffenen und die ordnungspolitische Vernunft einer Entscheidung. Insofern müssen Lagebeurteilungen und Entscheidungen ein hohes Maß an plausibler Kommunikationsfähigkeit besitzen. Im Ergebnis kann dies auch bedeuten, dass die Individuen berechtigte Interessen und Erwartungen zurückstellen müssen.

\section{WERTEORIENTIERUNG UND STRATEGIEDEBATTE AUF POLITIKFELDERN}

Die Gegenüberstellung von alter und neuer Wertetrias gibt uns ein Gesamtbild über die veränderte Großwetterlage. Aber sie gibt Zwischenstufen, Ungleichzeitigkeiten und Widersprüche nicht hinreichend wieder. Man muss über Politikfelder reden, um sich die zentralen Zielwidersprüche zu vergegenwärtigen, die die gewerkschaftspolitische Orientierungs- und Strategiedebatte prägen. Probleme und Perspektiven zeitgemäßer Wertepolitik skizzieren wir beispielhaft an den Zielen "gute Arbeit" und "soziale Sicherheit" sowie an den beiden aktuellen Themen „Gesundheitsreform“ und „Arbeit im Alter“.

\section{ES GIBT ZWEI FORMEN GUTER ARBEIT}

Im Ziel der „guten Arbeit“ vereinigen sich die Werte der Gewerkschaften fast auf ideale Art und Weise. Gute Arbeit entspricht den Freiheitsvorstellungen inner- und außerhalb der Arbeit. Sie umfasst die Mitbestimmung im Unternehmen ebenso wie die Freiheit, auf der Grundlage des Normalarbeitsverhältnisses das Leben gestalten zu können. Sie entspricht der Vorstellung von materieller und kultureller sozialer Gerechtigkeit und auch der Solidarität, wenn sie verallgemeinert werden kann. Gute Arbeit, das ist ein festes, verlässliches Einkommen, unbefristete Beschäftigung, Einbringen kreativer Fähigkeiten, Sinn, Anerkennung, soziale Beziehungen, Achtung und Schutz der Gesundheit.

Doch der generelle gesellschaftliche Trend geht in eine andere Richtung: Teilzeitarbeit, Leiharbeit, Ein-Euro-Jobs, Mini-Jobs, Zeitarbeit, Vorruhestand, Altersteilzeit, befristete Job, keine Jobs, prekäre und neue Arbeitsverhältnisse, Flexibilisierung der Arbeitszeiten, Niedriglöhne, Flächentarife, Haustarife, keine Tarife - die Pluralität von Arbeitsverhältnissen und -regelungen wächst. Mittlerweile haben die Mittelklassen, die klassischen Gewerkschaftsmitglieder, massive Abstiegsängste erfasst. Vielen älteren und einfach qualifizierten Menschen droht Nicht-Integration. Einem größer werdenden Teil der Bevölkerung im unteren Drittel der Gesellschaft fehlt jegliche Perspektive. Hier steht die Integrationsfähigkeit unserer Gesellschaft insgesamt auf dem Spiel. 
Es gibt zwei Formen guter Arbeit. Wenn für alle Qualifizierten Einkommen, Sinn und gesellschaftliche Anerkennung erfüllt werden, ist das eine Ziel guter Arbeit verwirklicht. Aber längst nicht alle, die gute Qualifikationen besitzen, haben eine gute unbefristete Stelle mit einem gesicherten Einkommen. Für freischaffende Kreative und neue Selbstständige haben die Gewerkschaften nun endlich Mindestbedingungen bei der Zeitarbeit ausgehandelt, nachdem diese Arbeit lange als „moderne Sklavenarbeit" verpönt war. Wenn sowohl die qualifizierten, aber prekär beschäftigten Arbeitnehmerinnen und Arbeitnehmer eine bessere arbeitsvertragliche Grundlage erhalten als auch den gering Qualifizierten die Möglichkeit zum Einstieg eröffnet wird, wird das zweite Ziel guter Arbeit erreicht. Für die sogenannten Risikogruppen des Arbeitsmarktes, die gering qualifizierten Menschen, die Berufseinsteiger, bedarf es eigener Teilhabeperspektiven, die nicht unbedingt alle dem Maßstab eines Normalarbeitsverhältnisses entsprechen müssen. Gewerkschaften sollten offensive Konzepte anbieten, wenn Teile der Bevölkerung keinen Anschluss finden. Die Ausgestaltung eines zweiten und dritten Arbeitsmarkts muss in Betracht gezogen werden.

\section{DAS ZIEL HEIßT FLEXIBLE SOZIALE SICHERHEIT}

Soziale Sicherheit und die Möglichkeiten positiver und negativer Freiheit sind unter Druck geraten. Wenn es keine absolute Sicherheit in modernen Gesellschaften geben kann, wird es zum Kennzeichen guter Politik, die Bedingungen für eine dynamische Sicherungskonstellation in dreierlei Hinsicht zu organisieren. Erstens müssen Risiken minimiert werden, die sich durch Krankheit, Unfälle, Alter, Verbrechen und Arbeitslosigkeit ergeben können. Zweitens geht es um eine Ungesichertheit, die durch das Fehlen sozialer Garantien für die materiellen, sozialen und kulturellen Existenzgrundlagen des Individuums entsteht. Drittens muss der Unsicherheit begegnet werden, die mit dem Wandel diskontinuierlicher Erwerbsbiografien einhergeht. Dafür müssen Brücken und Netze gebaut werden, um auf diese Ungewissheiten des neuen Berufslebens einzugehen.

Das Ziel heißt flexible soziale Sicherheit. Dafür können wir nicht in der 100jährigen Tradition der bismarckschen Politik stehen bleiben, sondern müssen die vor- handene Sicherheitsarchitektur weiter entwickeln. Ein vielversprechender Ansatz ist das Sicherungskonzept der „Flexicurity“, das von der Hans-Böckler-Stiftung für die deutsche Debatte aufgenommen wurde. "Flexicurity“ soll gestiegene Anforderungen an Flexibilität („Flexibility“) in den Arbeits- und Lebensverhältnissen mit neuen Formen der sozialen Sicherheit („Security") beantworten. Angestrebt wird eine Balance zwischen der Flexibilität auf dem Arbeitsmarkt und in der Arbeit einerseits und der Beschäftigungs- und sozialen Sicherheit andererseits. Dafür müssen die Einsatzmöglichkeiten eines umfänglichen Insrumentariums geprüft werden.

\section{SOLIDARITÄT UND EIGENVERANTWORTUNG}

Die Solidarität im Gesundheitswesen steht zur Disposition. Den Konservativen und Wirtschaftsliberalen ist es bislang noch immer gelungen, aus Eigenverantwortung und besserem Umgang mit den zur Verfügung stehenden Ressourcen einerseits und dem Gemeinwohl sowie dem Wert der Solidarität andererseits einen Gegensatz zu konstruieren. Aber Solidarität und Eigenverantwortung sind keine Gegensätze. Auf beide Werte kann in der Reformdebatte des Gesundheitssystems nicht verzichtet werden.

Die Konservativen und Wirtschaftsliberalen heben einseitig die Rolle der Eigenverantwortung im Sinne der Privatisierung von Risiken hervor, ohne die Pflichten der Anbieter zu thematisieren. Klassische Wertepolitik in der Gesundheitspolitik beschränkte sich darauf, alle unabhängig von ihrem Erwerbsstatus mitzuversichern, ohne nach der Eigenverantwortung zu fragen. Beide Positionen sind unhaltbar. Wer Ansprüche an Solidarität negiert, hat keinen moralischen Kredit, Eigenverantwortung einzufordern. Zugleich gilt es, den Solidargedanken um den Aspekt des eigenverantwortlichen Verhaltens zu erweitern. Die Versicherten müssen sich eigenverantwortlicher verhalten, um Solidarität erwarten zu können, aber die Anbieter im Gesundheitswesen müssen sich gleichzeitig solidarischer verhalten, indem sie ihre Sonderinteressen zugunsten des öffentlichen Gutes „Gesundheit" zurücknehmen. Auf der Angebotsseite müssen die Verantwortlichkeiten für ein solidarisches Gesundheitssystem klarer benannt werden. Auf der Nachfrageseite muss das Prinzip der Eigen- verantwortung, d.h. die Abschätzung der Gesundheitsrisiken der eigenen Lebensführung, befördert werden. Verantwortlich mit sich selbst umzugehen, heißt, Verantwortung für das Gesundheitswesen als Solidargemeinschaft zu übernehmen.

Die Gewerkschaften können in der anstehenden Reform des Gesundheitswesens die Verantwortung der Akteure mit Begriffen von Solidarität und Eigenverantwortlichkeit thematisieren: mehr Eigenverantwortung von Versicherten und Patienten, mehr Solidarität von Ärzten etc. Die Gewerkschaften können weiterhin, gemeinsam mit Betriebsräten, Arbeitgebern und den Unternehmen, die betriebliche Gesundheitspolitik forcieren und dabei auf Konzepte wie eine „Zukunftsfähige betriebliche Gesundheitspolitik“ zurückgreifen. Wenn Betriebe, Sozialpartner, Staat, Sozialleistungsträger sowie Bildung und Wissenschaft sich daran beteiligen, werden sie ihrer Verantwortung für Solidarität gerecht.

\section{FLEXIBLE ALTERSGRENZEN STATT STARRER REGULIERUNG}

Angesichts der demografischen Entwicklung in Deutschland ist es erforderlich, erweiterte Teilhabemöglichkeiten für Ältere an der Arbeit zu schaffen. Die Altersteilzeit war ursprünglich Vehikel für Solidarität und positive Freiheit. In einer Zeit knapper Arbeitsplätze für junge qualifizierte Menschen und ausreichender sozialstaatlicher Ressourcen war es eine Idee solidarischen Verhaltens, dass Ältere für Junge Platz machten und in den Vorruhestand gingen. Es war gleichzeitig auch ein Akt der Befreiung von Jahrzehnte langer Erwerbsarbeit und die Möglichkeit, privaten Interessen und Neigungen nachgehen zu können.

Heute müssen die Weichen für eine alters- und alternsgerechte Gestaltung der Arbeit gestellt werden. Flexible Altersgrenzen sind dafür angemessener als starre Regulierungen, die wenig mit der individuellen Konstitution und persönlichen Optionen zu tun haben. Mehr Wahlmöglichkeiten lassen sich auch durch eine bessere Balance zwischen Arbeit und Leben, zwischen Beruf und Familie schaffen. Daran haben viele Menschen großes Interesse. Deshalb muss es gelingen, zu praktischen betrieblichen oder unternehmensbezogenen Vereinbarungen zu gelangen.

Nicht nur für die Einstellung von Arbeitskräften, sondern auch für die Ent- 
wicklung alterns- und altersgerechter Arbeits-, Leistungs- und Qualifikationsbedingungen kommt dem Management eine herausragende Rolle zu. Festgestellt werden kann derzeit ein Mangel an längerfristig angelegten Personalstrategien, die auf der Integration von Arbeitsorganisation, Qualifizierung und Gesundheitspolitik aufbauen. Stattdessen dominiert in den meisten Unternehmen nach wie vor eine Statusquo-Politik, die auf junge Belegschaften setzt, wobei den Älteren häufig bestenfalls die Rolle des flexiblen Personalpuffers eingeräumt wird.

Arbeitspolitik muss sich auf eine umfassendere Gestaltung des demografischen Wandels einlassen. Dabei geht es nicht nur um eine „Politik zugunsten älterer Beschäftigter", sondern um eine Politik, die Ansätze wie „altersgemischte Belegschaften“, den betrieblich organisierten Wissenstransfers zwischen Jung und Alt, das lebensbegleitende Lernen inklusive einer neuen Weiterbildungskultur und präventive Gesundheitsaktivitäten integriert.

\section{AM ENDE BEIDES: INTERESSENBEZUG UND UNIVERSELLER ANSPRUCH}

Gerade für Gewerkschaften ist es wichtig, sich der Wertbezogenheit ihres Handelns $\mathrm{zu}$ vergewissern und dieses zu kommunizieren. Vorhandene Werte müssen weniger bejaht als vielmehr zeitgemäß interpretiert werden, und zwar im Lichte der Arbeitsund Lebensbedingungen der auf abhängige Arbeit angewiesenen Menschen. Eines muss deutlich werden: Es gibt eine Wertgebundenheit des Handelns, die Basis und Legitimation von gewerkschaftlichen Zielen und Instrumenten ist. Letztlich sind es nicht die Werte an sich, die es den Gewerkschaften ermöglichen, sich von anderen Interessen abzugrenzen. Es ist vielmehr das spezifische Zusammenspiel zwischen Werten, Zielen und Instrumenten. Und diese Verbindung können die Gewerkschaften so gestalten, dass sie neben einem klaren Bezug auf die spezifischen Interessen ihrer Mitglieder auch einen universellen Anspruch verfolgen.

\section{Ein Schritt auf dem Weg aus der Defensive? Die Tarifrunde 2007 in der Metall- und Elektroindustrie}

\section{LEBEN IN UND WEGE AUS DER DEFENSIVE}

Dass sich die Gewerkschaften in den entwickelten kapitalistischen Gesellschaften in einer strukturellen Defensive befinden, gilt mittlerweile in Wissenschaft und Politik als Allgemeinplatz. Durchaus nicht zu Unrecht. Mitgliederzahlen und Finanzressourcen der Gewerkschaften sind rückläufig, die verteilungspolitischen Anteile der Lohnabhängigen bleiben hinter den besitzstandsneutralen Verteilungsspielräumen aus Inflation und Produktivität zurück, und auf betrieblicher Ebene unterliegen Belegschaften, betriebliche Interessenvertretungen und Gewerkschaften ein um das andere Mal dem Erpressungsdruck von Konzernleitungen. Hinzu kommt - nicht nur in Deutschland - ein Zerwürfnis zwischen Gewerkschaften und den sozialdemokratischen bzw. sozialistischen Parteien und damit einhergehend eine Schwächung der Repräsentanz gewerkschaftlicher Forderungen in den politischen Arenen. Alles in allem keine komfortable Situation für die Gewerkschaften.

Doch neben diesen Anzeichen einer realen Krise sind in jüngster Vergangenheit auch Indikatoren für eine Konsolidierung der Gewerkschaften sichtbar geworden. Einigen Gewerkschaften gelang eine deutliche Verlangsamung des Mitgliederschwundes, und die finanzielle Basis konnte stabilisiert werden. Zunehmend enden betriebliche Kämpfe um Standorte, Arbeitsplätze und Sozialstandards wenn nicht mit dem Erhalt der Arbeitsplätze, so doch mit erheblichen Verbesserungen der sozialen Absicherung der Betroffenen. Und auch die diesjährigen Tarifabschlüsse in der Chemieund Bauindustrie signalisieren eine, wenn auch vorerst noch bescheidene, Rückkehr gewerkschaftlicher Verhandlungsmacht.

Zweifelsohne beruhen gewerkschaftliche Konsolidierungserfolge auch auf dem in dieser Vehemenz nicht erwarteten ökonomischen Aufschwung. Mit ihm hat sich die Druckempfindlichkeit der Arbeitgeber erhöht und die Verhandlungsposition der
Gewerkschaften verbessert. Doch zugleich sollte nicht ausgeschlossen werden, dass die Anzeichen einer Konsolidierung der Gewerkschaften auch auf die zunehmende Fähigkeit verweisen könnten, sich besser als in der Vergangenheit auf die strukturellen Herausforderungen der gegenwärtigen gesellschaftlichen Umbrüche einzustellen.

An diesen Indizien für eine gesellschaftliche Konsolidierung der Gewerkschaften und ihren Hintergründen setzt eine wissenschaftliche Debatte an, die im Begriff ist, sich unter dem Label „Labor Revitalization Studies“ bzw. „Strategic Unionism“ zu einem Forschungszweig zu

Christoph Ehlscheid, Ressortleiter Strategi-
sche Planung beim Vorstand der IG Metall.
e-mail: christoph.ehlscheid@igmetall.de
Hans-Jürgen Urban, Dr., Leiter des Funktions-
bereichs Gesellschaftspolitik/Grundsatz-
fragen/Strategische Planung beim Vorstand
der IG Metall.
e-mail: hans-juergen.urban@igmetall.de

Christoph Ehlscheid, Ressortleiter Strategische Planung beim Vorstand der IG Metall. e-mail: christoph.ehlscheid@igmetall.de Hans-Jürgen Urban, Dr., Leiter des Funktionsfragen/Strategische Planung beim Vorstand e-mail: hans-juergen.urban@igmetall.de 
konstituieren. ${ }^{1}$ Ohne die Dimension der gewerkschaftlichen Defensive zu ignorieren, wendet sich dieser Ansatz insbesondere gegen die implizite Zwangsläufigkeit diverser Niedergangsprognosen und betont Möglichkeiten und Verantwortung der Gewerkschaften für die Realisierung vorhandener, positiver Strategieoptionen. Um diese zu identifizieren, wird der wissenschaftliche Fokus verschoben: Anstatt die gewerkschaftliche Krise (nur) auszuleuchten, wird nach den Voraussetzungen einer gewerkschaftlichen Revitalisierung und einer verbesserten gewerkschaftlichen Strategiefähigkeit gefragt.

Auch wenn der Begriff der „gewerkschaftlichen Revitalisierung " in dieser Debatte mitunter unscharf bleibt, im Kern zielt er auf eine organisationspolitische Stabilisierung und die Erhöhung der gewerkschaftlichen Durchsetzungsfähigkeit in den Aushandlungs- und Verteilungskonflikten mit Unternehmen und Staat. Systematisch können dabei vier Dimensionen der Erneuerung gewerkschaftlicher Organisations- und Verhandlungsmacht und damit einer erfolgreichen bzw. erfolglosen Revitalisierung unterschieden werden ${ }^{2}$. Erstens: Die „Mitglieder-Dimension“ („membership"), die nach der Entwicklung der Mitgliederzahlen, dem Organisationsgrad und der Übereinstimmung der sozialen Mitgliederstruktur mit der gesellschaftlichen Sozialstruktur als Voraussetzungen gewerkschaftlicher Organisationsmacht fragt; zweitens: die ökonomische bzw. die verhandlungspolitische Dimension („economic and bargaining power"), die vor allem auf die kollektive Verhandlungsmacht und die Fähigkeit zu Lohn- und Sozialabschlüssen abhebt; drittens: die politische Dimension („political power“), die die gewerkschaftliche Lobbymacht im Sinne des Einflusses auf staatliche Entscheidungen bewertet; und schließlich viertens: die institutionelle Dimension (,institutional vitality"), die den Fokus auf die interne Anpassungs-, Strategie- und Führungsfähigkeit der Gewerkschaften richtet.

Ohne an dieser Stelle auf theoretische Prämissen, forschungspolitische Implikationen und konzeptionelle Probleme dieses Ansatzes einzugehen, soll im Folgenden gefragt werden, ob aus der Tarifrunde 2007 in der Metall- und Elektroindustrie Rückschlüsse auf den Revitalisierungsstand der deutschen Gewerkschaften, genauer: der IG Metall gezogen werden können. Anders ausgedrückt, es soll diskutiert werden, ob dieser Verteilungskonflikt als ein Beitrag zur Stärkung der gewerkschaftlichen Verhandlungs- und Verteilungsmacht und damit als ein Schritt in Richtung einer Revitalisierung der IG Metall gewertet werden kann.

\section{DIE ECKPUNKTE DES TARIFABSCHLUSSES}

Das mediale Echo auf die Tarifrunde scheint zunächst eine positive Antwort auf die Frage nach einem Konsolidierungsbeitrag dieser Tarifrunde für die IG Metall nahezulegen. Sowohl in der Boulevardpresse als auch in den Berichten des Flaggschiffs des neoliberalen Wirtschaftsjournalismus, der „Frankfurter Allgemeinen Zeitung“, ging diese Runde an die IG Metall. Während die „Bild“-Zeitung die angeblich höchste Lohnerhöhung seit 15 Jahren geradezu bejubelte, diagnostizierte die FAZ mürrisch einen „Sieg der IG Metall“. Doch hält diese schnelle Bewertung auch einer Überprüfung stand, welche die vielfältigen politischen Implikationen des Abschlusses mit einbezieht?

Zunächst zu den zentralen Ergebnissen des Tarifabschlusses: Für die Anpassung der Entgelte wurde eine mehrstufige Regelung getroffen. Für die Monate April und Mai 2007 erhalten die Beschäftigten eine einmalige Pauschalzahlung von $400 €$ und die Auszubildenden eine Pauschale von $125 €$. Dem folgt eine tabellenwirksame Erhöhung für die nächsten zwölf Monate von 4,1\%, wobei die Ausbildungsvergütungen durch ihre Anbindung an die Entgelttabellen oder gesonderte Regelungen entsprechend erhöht werden. Für die Monate Juni 2008 bis November 2008 wurden eine weitere tabellenwirksame Entgelterhöhung von 1,7 \% sowie eine Einmalzahlung in Höhe von fast $4 \%$ eines durchschnittlichen Monatseinkommens vereinbart. Den Betriebsparteien steht jedoch auf der Grundlage einer freiwilligen Betriebsvereinbarung die Möglichkeit offen, die zweite Entgelterhöhung sowie die Einmalzahlung bis spätestens 1 . Oktober 2008, also für einen Zeitraum von vier Monaten, zu verschieben.

Neben den Entgelterhöhungen wurde auch eine „Vereinbarung zur Weiterentwicklung der Tarifregelungen zum flexiblen Übergang in die Rente" getroffen. Hintergrund sind die jüngeren Auseinandersetzungen um die Rente mit 67 bzw. die auslaufenden Regelungen für die Altersteilzeit. In der Vereinbarung erklären die Tarifvertragsparteien ihre gemeinsame Auffassung, dass aufgrund der demografischen
Entwicklung und der gesetzlichen Regelungen ein überregionaler gesetzlicher wie tarifvertraglicher Handlungsbedarf bestehe, damit die Betriebe auf die Herausforderungen einer "demografiefesten Personalpolitik" reagieren können.

\section{EIN GEWERKSCHAFTLICHER ERFOLG...}

Aus einer gewerkschaftlichen, organisationspolitischen Sicht kann die gesamte Tarifrunde 2007 als eindeutiger Erfolg gewertet werden. Die Diskussion um eine geeignete Tarifforderung konnte breit und beteiligungsorientiert geführt werden. Die gemeinsam erstellte Forderung nach einer Einkommensverbesserung von $6,5 \%$ traf in der Mitgliedschaft auf hohe Akzeptanz. Auch die Verhandlungsphase war in einen hohen innergewerkschaftlichen Konsens eingebettet und verlief unter breiter Einbeziehung der Belegschaften. Obwohl früh klar wurde, dass einige Tarifbezirke keine „Abschlussbezirke“ sein würden, verabschiedeten sich diese nicht aus der Tarifrunde. Die außerordentlich hohe, bundesweite Mobilisierungsbereitschaft der Belegschaften machte deutlich, dass die Tarifbewegung von Anfang bis Ende von der gesamten Organisation getragen wurde. Mit einer Beteiligung von über 300.000 Beschäftigten erreichte die Warnstreikwelle am Tag vor der entscheidenden Verhandlungsrunde ein in der Sozialgeschichte der Bundesrepublik einmaliges Beteiligungsniveau. Auch das Ergebnis übertraf in weiten Bereichen die Erwartungen der Mitglieder. Mit seiner durchgängig hohen Akzeptanz dürfte es zu einer Festigung der Bindung der Mitglieder geführt haben.

Schwerer fällt die verteilungspolitische Bewertung des Tarifabschlusses. Zunächst ist der erste Teil des Abschlusses (Pauschale von $400 €$ und $4,1 \%$ ) ein beachtlicher verteilungspolitischer Erfolg. Insbesondere die Einmalzahlung bedeutet für die Einkommen unterhalb der „Eckentgelte“ (Ent-

\footnotetext{
1 Frege, C./Kelly, J. (2004): Varieties of Unionism: Strategies for Union Revitalization in a Globalizing Economy, Oxford. Urban, H.-J. (2006): Vom Krisen- zum Strategieparadigma? Argumente für Nutzen und Möglichkeit einer Neuausrichtung der Gewerkschaftsforschung. Vortrag an der Universität Jena, (Manuskript). AG Strategic Unionism (2007): Aus der Krise zur Erneuerung? Umrisse eines Forschungsprogramms (Manuskript), Jena.

2 Dazu insbesondere Frege, C./Kelly, J. (2004): a.a.O
} 
gelte für Facharbeiten) eine zweimonatige Erhöhung zwischen 8 und $12 \%$. Diese überproportionale Anhebung unterer Einkommen stellt eine ausgeprägte „soziale Komponente" dar und rückt diesen Teil des Abschlusses in die Nähe einer offensiven „solidarischen Lohnpolitik“. ${ }^{3}$ Zugleich erhöht sie das Verteilungsvolumen für die ersten 14 Monate deutlich über die $4 \%$ und realisiert damit eine ebenfalls deutliche Umverteilungskomponente, die aus Konjunktur- wie Gerechtigkeitsgründen eindeutig zu begrüßen ist.

\section{...MIT RISIKOPOTENZIAL}

Problematischer ist dagegen der zweite Teil des Abschlusses. Die Option auf eine viermonatige Verschiebung der zweiten Erhöhungsstufe stellt eine Art ungedeckten Scheck auf die verteilungspolitische $\mathrm{Zu}$ kunft dar. Ihr wohnt das Risiko einer verteilungspolitischen Ex-Post-Entwertung des gesamten Abschlusses inne. Sie könnte zur Falltür werden, die eine erhebliche Gefahr für die Verteilungsposition der Beschäftigten darstellt. Zugleich unterscheidet sich diese Option von der im Tarifvertrag 2006 vorgesehenen Flexibilisierungsregelung durch ihre asymmetrische Struktur. Im Gegensatz zur letztjährigen $310 €$-Regelung sieht die jetzige Vereinbarung keine Flexibilitätsspielräume nach oben und unten, sondern nur nach unten vor. ${ }^{4}$ So kann zwar die anstehende Tariferhöhung um vier Monate nach hinten geschoben, nicht aber vorgezogen werden. Sie pendelt damit also nicht um einen tariflich fixierten Zeitpunkt, sondern sieht lediglich eine Abweichungsmöglichkeit nach unten vor. Das bedeutet: Sie ist kein Flexibilitätselement im eigentlichen Sinne, sondern eher eine Unterbietungsoption. Die Anwendung dieser Regelung wird letztlich über die verteilungspolitische Substanz des gesamten Abschlusses entscheiden. Würde die zweite Stufe der Entgelterhöhung um volle vier Monate ausgesetzt, entwertet das nicht nur die verteilungspolitisch erfolgreiche erste Stufe. Da nicht damit zu rechnen ist, dass sich die gute Konjunktur in der Metall- und Elektroindustrie im Jahr 2008 merklich abschwächt, würde sie darüber hinaus die ohnehin nicht sehr deutliche Entgelterhöhung in der zweiten Phase weiter reduzieren. Die Verteilungsposition der Lohnabhängigen würde sich weiter verschlechtern.

Das Flexibilisierungselement prägt auch die ordnungspolitische Bewertung des Ab- schlusses. Bereits vorherige Abschlüsse haben das tradierte Verhältnis zwischen Flächentarifvertrag und betrieblichen Regelungsoptionen neu justiert. Dabei geht der Trend eindeutig in Richtung einer Verlagerung von Regelungsinhalten und Entscheidungskompetenzen von der flächenvertraglichen auf die betriebliche Handlungsebene. ${ }^{5}$ Auch der aktuelle Tarifvertrag folgt dieser Tendenz. Damit können die Betriebsparteien direkt in die tarifvertragliche Kernsubstanz eingreifen, und sie erhalten eine gegenüber bisherigen Regelungen erweiterte Entscheidungskompetenz. Während es prosperierenden Unternehmen mit durchsetzungsstarken Betriebsräten und mobilisierungsbereiten Belegschaften gelingen dürfte, Forderungen der Arbeitgeberseite erfolgreich entgegenzutreten, ist das Risiko abgepresster verteilungspolitischer Zugeständnisse in Krisenbranchen und -unternehmen mit verängstigten Belegschaften und in die Defensive gedrückten Interessenvertretungen höher.

Gleichwohl würde es auf ein verzerrtes Bild dieser Tarifrunde hinauslaufen, würden nicht auch die risikomindernden Faktoren in die Betrachtung einbezogen. So stärken die guten Konjunkturaussichten die Verhandlungsposition der betrieblichen Interessenvertretungen und verbessern damit die Voraussetzungen für die betriebspolitische Verteidigung tariflich errungener Verteilungspositionen. Zudem sind die rechtlichen Regelungen eher zugunsten der Beschäftigten ausgestaltet. Eine Abweichung nach unten ist lediglich auf der Grundlage einer freiwilligen Betriebsvereinbarung zwischen den Betriebsparteien - also nur nach einer aktiven Zustimmung der Betriebsräte - möglich. Es spricht also einiges dafür, dass die verteilungspolitischen Risiken des Abschlusses zu bewältigen sind und die entsprechenden Regelungen sich eher als mittelfristige ordnungspolitische Herausforderung denn als aktuelles verteilungspolitisches Problem erweisen werden.

\section{ZEIT FÜR EINE GEWERKSCHAFTS- UND TARIFPOLITISCHE STRATEGIE- DEBATTE}

Gleichwohl wäre die IG Metall gut beraten, die ordnungspolitischen Implikationen des Abschlusses in eine ohnehin anstehende gewerkschafts- und tarifpolitische Strategiedebatte einzubeziehen. Eine umfassende Bewertung des Tarifabschlusses in der Metall- und Elektroindustrie fällt also diffe- renziert aus. Organisationspolitisch kann die IG Metall einen klaren Erfolg verbuchen, und auch für die ersten 14 Monate ist der verteilungspolitische Gehalt der Tarifvereinbarung durchaus beeindruckend. Doch die verteilungspolitischen und ordnungspolitischen Risiken sollten keineswegs übersehen werden. Ob die potenziellen Erfolge dieser Runde gesichert und die Risiken abgewehrt werden können, hängt nicht zuletzt von der Fähigkeit der IG Metall ab, gerade in den Kernfeldern der gewerkschaftlichen Betriebs- und Tarifpolitik eine Reihe strategischer Schlüsselaufgaben erfolgreich zu bearbeiten.

\section{ZUM ZUKÜNFTIGEN VERHÄLTNIS VON BETRIEB UND FLÄCHEN- TARIFVERTRAG}

Auch das Ergebnis der diesjährigen Tarifrunde ist Teil einer strukturellen Entwicklung, die als Umbruch in der deutschen Tariflandschaft und Erosion des Flächentarifvertragssystems beschrieben wird. Neben dem merklichen Rückgang der Tarifbindung infolge der Austritte aus den Arbeitgeberverbänden oder des Wechsels in Arbeitgeberverbände ohne tarifverbindliche Wirkungen (OT-Verbände) ist eine systematische Entwertung von Kerngehalten und Verbindlichkeit flächentarifvertraglicher Regulierungen zu beobachten. Diese findet im Zuge einer umfassenden Dezentralisierung bzw. Verbetrieblichung der Tarifpolitik statt. Sie wird tarifrechtlich durch eine massive Ausbreitung von Öffnungsklauseln durchgesetzt, die betriebliche Abweichungen von überbetrieblich festgelegten Standards ermöglichen. ${ }^{6}$ Hinzu kommt eine wachsende Differenzierung von Tarif-

3 Schulten, Th. (2004): Solidarische Lohnpolitik in Europa. Zur Politischen Ökonomie der Gewerkschaften, Hamburg.

4 Zur Bewertung des Tarifergebnisses:2006: Ehlscheid, Ch./Urban, H.-J. (2006): Tarifpolitische Weichenstellung, in: Blätter für deutsche und internationale Politik 6, S. 655-657.

5 WSI in der Hans-Böckler-Stiftung (Hrsg.) (2006): Tarifhandbuch, Abschied vom Flächentarifvertrag? Das deutsche Tarifsystem im Umbruch, Frankfurt/M.

6 Für den Prozess der Verbetrieblichung lassen sich vier Etappen identifizieren; vgl. dazu ausführlich Bispinck, R./Bahnmüller, R. (2007): Abschied vom Flächentarifvertrag? Der Umbruch in der deutschen Tariflandschaft und seine Konsequenzen für eine betriebsorientierte Tarifpolitik, in: Bispinck, R. (2007): Wohin treibt das Tarifsystem?, Hamburg S. 9-28. 
standards nach Beschäftigtengruppen und Tätigkeitsbereichen, die sich zum Teil über die Anwendung von tariflichen Öffnungsklauseln oder über die Vereinbarung zusätzlicher Abkommen für spezifische Beschäftigtengruppen (z. B. im industrienahen Dienstleistungsbereich) vollzieht. Aber nicht nur die Herabsetzung der Verbindlichkeit tarifvertraglicher Normen wird vorangetrieben. Zum strategischen Arsenal der Arbeitgeberverbände gehört auch die Stärkung und „Umnutzung“ tariflicher Normen. Im Rahmen dieser Strategie kommt es zu einer Neudefinition der Funktion von Tarifverträgen. Galten sie bisher als Instrumente des Schutzes und der Durchsetzung der Interessen der abhängig Beschäftigten, so sollen sie nun unter gezielter Nutzung der Ordnungsund Befriedungsfunktion - zu Instrumenten der Infragestellung und Reduzierung erreichter Tarifstandards mutieren. ${ }^{7}$

In dieser neuen tarifpolitischen Strategie werden die Akzente verschoben: „Sie beinhaltet die Umnutzung von Tarifverträgen zu einem Instrument der Durchsetzung von Arbeitgeberinteressen, ein nachdrückliches Insistieren auf der Ordnungsfunktion von Tarifverträgen, wo diese nützlich erscheint, und eben auch die Herabsetzung bzw. Auflösung der Verbindlichkeit von Tarifnormen durch Verlagerung der Regelungskompetenz auf die betriebliche Ebene." 8 Durch die Kombination aus Verbetrieblichung, Flexibilisierung und Umnutzung flächentarifvertraglicher Normen sollen Verbindlichkeit und Kostenbelastungen von Tarifverträgen reduziert werden, ohne auf die ordnungspolitischen und disziplinierenden Funktionen von Tarifverträgen verzichten zu müssen. In einer solchen Konzeption werden Tarifstandards nicht mehr als Mindestnormen angesehen, sondern zur Höchstnorm, deren Überschreitung vermieden werden soll, während die Unterschreitung jederzeit möglich sein muss.

Es ist nicht zu erkennen, dass die Gewerkschaften die Strategievarianten im Arbeitgeberlager bereits hinreichend analysiert und ihrerseits adäquate Antworten entwickelt hätten. Weder verfügen sie bisher über ein einigermaßen konsistentes Konzept, in dem das Verhältnis zwischen der betrieblichen und der flächentarifvertraglichen Regelungsebene effektiv austariert ist, noch scheinen sie machtpolitisch ausreichend auf das umfassende tarifpolitische Roll-Back der Arbeitgeberverbände vorbereitet zu sein. Sowohl das „Pforzheimer Abkommen“ aus dem Jahre 2004 als auch die diesjährige Übertragung der verteilungspolitischen Funktion des Tarifvertrages an die Betriebsparteien waren keine bewusst anvisierten Elemente einer gewerkschaftlichen Strategie. Sie wurden eher aus der Not der zugespitzten verhandlungspolitischen Situation heraus geboren.

\section{AUS DER NOT EINE TUGEND MACHEN}

Das eigentliche Ziel der Verbetrieblichung der Tarifpolitik besteht aus Arbeitgebersicht in einer Verschiebung der machtpolitischen Verhandlungspositionen zu eigenen Gunsten. Denn während sich die IG Metall in kollektiven überbetrieblichen Tarifrunden immer noch als handlungsfähiger Akteur profiliert, fällt es einzelnen Belegschaften und ihren Interessenvertretungen angesichts von Massenarbeitslosigkeit, Standortkonkurrenz und betrieblichen Rationalisierungsstrategien in der Regel schwerer, ihre Interessen gegenüber der Unternehmensleitung durchzusetzen. Die Verlagerung von Regelungskompetenzen auf die betriebliche Ebene geht üblicherweise mit einer Verlagerung von Machtressourcen auf die Arbeitgeberseite einher. Seit geraumer Zeit jedoch suchen die Gewerkschaften nach angemessenen Antworten. Zunehmend werden strategische Ansätze entwickelt und praktiziert, die durch eine Neujustierung von Tarif- und Betriebspolitik der machtpolitischen Unterlegenheit entgegenwirken sollen. In diesem Sinne wird die Infragestellung tarifvertraglicher Normen in den Unternehmen als Anlass organisierter und koordinierter Gegenwehr der Belegschaften genutzt. Im Zuge beteiligungs- und mobilisierungsorientierter Gegenwehrkonzepte soll aus der Not der Verbetrieblichung der Tarifverträge die Tugend einer über Tariffragen organisierten Politisierung der gewerkschaftlichen Betriebsarbeit gemacht werden. Dem kollektiven Kampf um Tarifstandards in allgemeinen Tarifrunden folgt eine „zweite Tarifrunde", die diesmal auf betrieblicher Ebene ausgetragen wird und der Sicherung der erkämpften Tarifstandards dient.

Eine solche betriebspolitische Neufundierung der Tarifpolitik kann die Identifikation der Belegschaften mit zuvor erkämpften Tarifinhalten erhöhen und zugleich die Mobilisierungskraft der Gewerk- schaften in den Betrieben ausbauen. „Die Tarifparteien agieren zunehmend auf der betrieblichen Ebene, und die Betriebsparteien verhandeln über Tarifmaterie. Mit der Neujustierung des Verhältnisses von Tarifund Betriebspolitik soll dieser Entwicklung Rechnung getragen und sie in eine Strategie umgemünzt werden, mit der die Gewerkschaften Stärke und Einfluss zurückgewinnen können. “9 In diesen Versuchen, durch eine politisierte Betriebsarbeit der Erosion des Flächentarifvertrages entgegenzuwirken und zugleich die gewerkschaftliche Durchsetzungs- und Mobilisierungskraft zu stärken, dürften Revitalisierungspotenziale für die Gewerkschaften liegen, die bisher nicht ansatzweise ausgeschöpft sind.

\section{QUALITATIVE ELEMENTE}

Neben den geschilderten Anforderungen an eine Neujustierung von gewerkschaftlicher Betriebs- und Tarifpolitik steht die IG Metall vor Herausforderungen, die aus dem gegenwärtigen rasanten Formwandel der Arbeit resultieren. Dieser vollzieht sich im Rahmen einer Unternehmenspolitik, die die Steuerung der Unternehmen am Börsenwert des Unternehmens und an den Renditeerwartungen der Finanzmärkte ausrichtet und die Abläufe so organisiert, dass der Marktdruck unmittelbar an die Beschäftigten weitergegeben wird. In dieser Strategie werden die an den Finanzmärkten orientierten Eigenkapitalrenditen und der für die Börsenbewertung relevante Cash flow als fixe, externe Größe in die Unternehmensplanungen integriert. Löhne, Arbeitszeiten, Arbeitsbedingungen werden ebenso zu abgeleiteten Restgrößen wie die Mitbestimmungs- und Arbeitsrechte der Beschäftigten und Betriebsräte.

\footnotetext{
7 Deutlich sichtbar wird diese Variante an dem Umgang der Arbeitgeber mit dem neuen Entgeltrahmenabkommen in der Metall- und Elektroindustrie. Bei der betrieblichen Umsetzung hat insbesondere Südwestmetall eine Strategie gewählt, die sich scheinbar positiv auf den Tarifvertrag bezieht, die Neueingruppierung als regelungstechnischen Vorgang begreift und Verteilungspolitik im Namen von Ordnungspolitik betreibt. Vgl. dazu Bahnmüller, R./Schmidt, W. (2006): Interesse, Strategie und Zielsetzungen der Tarifparteien im Umsetzungsprozess des ERA in Baden-Württemberg Erste Einschätzungen und Befunde. Arbeitspapier für den wissenschaftlichen Projektbeirat bei der Hans-Böckler-Stiftung sowie dies. in diesem Heft. 8 Ebd. S. 14.

9 Bispinck, R./Bahnmüller, R. (2006), a.a. O., S. 26.
} 
Im Zuge dieser Entwicklung wird das traditionelle Regime der Regulierung von Arbeit mit seinen Normen, die für die Begrenzung der Nutzung des menschlichen Arbeitsvermögens gesorgt hatten, schrittweise aufgebrochen. Das ist sowohl auf die „Arbeitszeit, den Arbeitsort und die Arbeitsleistung bezogen als auch auf die gesamten institutionellen Sicherungssysteme, die in der Vergangenheit das Normalarbeitsverhältnis (...) begründet haben". 10 Die negativen Folgen dieser Politik der „Entgrenzung“ sind nicht zu übersehen: Im Nonstop-Programm einer „Rund-umdie-Uhr-Gesellschaft“ bestimmen „Arbeiten ohne Ende", Stress und Arbeitshetze den Arbeitsalltag. Zudem gibt es einen ausgeprägten Trend zur Prekarisierung von Beschäftigungs- und Lebensverhältnissen. Ungeschützte und schlecht bezahlte Arbeitsverhältnisse mit Befristungen, als Leiharbeit und Scheinselbstständigkeit nehmen $\mathrm{zu}$ und generieren Soziallagen und Mentalitäten, die durch eine „Rückkehr der Unsicherheit" (Robert Castel) geprägt sind.

Auf diese Entwicklungen muss die gewerkschaftliche Arbeitspolitik reagieren, will sie die Gestaltung von Arbeit nicht den neuen Unternehmensstrategien und den vermarktlichten Machtstrukturen in den Unternehmen überlassen, sondern weiterhin relevanter Akteur der Regulierung der Arbeitsbedingungen bleiben. Das gilt nicht zuletzt für die IG Metall, die sich in der Vergangenheit durch die Vereinbarung von Mantel- und Lohnrahmentarifverträgen und ihre betriebliche Umsetzung im Verbund mit den Betriebsräten politisch profilieren und Ansehen bei den Beschäftigten erwerben konnte. Will die IG Metall dieses Profil nicht verlieren, so ist sie gezwungen, erneut qualitative Themen einer regulativen Arbeitspolitik auf die tarifpolitische Tagesordnung zu setzen, die der Entgrenzung von Arbeitszeiten und Leistungsanforderungen Grenzen setzt und moderne Industrie- und Dienstleistungsarbeit im Sinne menschengerechter Arbeits- und Leistungsbedingungen reguliert. ${ }^{11}$

Dass eine solche Strategie nicht nur von den Mitgliedern goutiert wird, sondern zugleich mit einer positiven Profilierung in der öffentlichen Debatte einhergehen kann, zeigte die Tarifrunde 2006, in der die IG Metall einen Tarifvertrag zur Qualifizierung durchsetzen konnte. Klar sollte aber auch sein, dass es mit einer schlichten Erweiterung der tarifpolitischen Agenda um neue, qualitative Themen nicht getan ist.
Ebenso wichtig wie die Debatte darüber, wie zeitgemäße tarifliche Regelungen aussehen könnten und unter welchen Voraussetzungen sie eine Chance auf Durchsetzung haben, ist die Frage, wie neue Regelungselemente betrieblich umgesetzt werden können und ihnen im Arbeitsalltag Geltung verschafft werden kann. Dies kann nicht im Sinne klassischer Stellvertreterpolitik geschehen: Ohne den aktiven Einsatz der Beschäftigten für „ihre Arbeitsbedingungen "läuft eine solche Arbeitspolitik ins Leere. Eine erfolgreiche „Grenzziehung “ ist deshalb nicht allein ein regelungstechnischer Vorgang, sondern eine Frage der Politisierung. Anknüpfungspunkte bietet das Projekt „Gute Arbeit“, mit dem die IG Metall konzeptionell und im betrieblichen Praxistest neue Wege ausgelotet hat. ${ }^{12}$

\section{ZUR REKONSTRUKTION EINER SOLIDARISCHEN LOHNPOLITIK}

Schließlich stünde eine Debatte über den verteilungspolitischen Anspruch der gewerkschaftlichen Tarifpolitik an. Die verteilungspolitische Bilanz der letzten Jahre enthält Elemente einer Chronik der gewerkschaftlichen Defensive. Angesichts der sinkenden Lohnquote, in der die wachsende Umverteilung zugunsten der Kapitaleinkommen zum Ausdruck kommt, und der steigenden Lohnspreizung zwischen den Beschäftigtengruppen ${ }^{13}$ lässt sich jedenfalls die Krise der „solidarischen Lohnpolitik“ kaum leugnen.

Noch in den Zeiten des korporatistischen „Modell Deutschland“ orientierten sich einige Gewerkschaften, unter ihnen die IG Metall, am Konzept einer solidarischen Lohnpolitik. Sie zielte zum einen auf eine offensive Verteilung zwischen Kapital und Arbeit zugunsten der Arbeitseinkommen. Zum anderen wollte sie eine möglichst egalitäre Verteilung zwischen den abhängig Beschäftigten erreichen und damit die Lohnspreizung möglichst gering halten. Nicht zuletzt aus der Perspektive einer Revitalisierung der Gewerkschaften scheint eine Debatte über die Chancen einer Rekonstruktion einer solidarischen Lohnpolitik geboten. Neben den positiven konjunktur- und wachstumspolitischen Effekten, die von einer besonderen Berücksichtigung der unteren Einkommensgruppen im Rahmen tariflicher Verteilungskonflikte zu erwarten wären, dürfte die „Verteilungswirkung in der Klasse“, die von einer egalitäreren Lohnpolitik ausgeht, zur positiven Profilierung der Gewerkschaften als einer Kraft der Propagierung von Gerechtigkeit in einer Gesellschaft beitragen, die zunehmend als ungerecht empfunden wird. Eine solche Thematisierung der normativen Aspekte tariflicher Verteilungspolitik würde zugleich die Brücke zur aktuellen Debatte um einen Mindestlohn schlagen und wäre anschlussfähig an die Diskussion über geeignete gesellschaftliche Strategien einer Entprekarisierung der Erwerbsarbeit.

Eine Schlüsselstellung in einem Konzept der Rekonstruktion einer solidarischen Tarifpolitik käme der Frage der lohnund tarifpolitischen Koordinierung zwischen den Einzelgewerkschaften des DGB zu. Während sich das Arbeitgeberlager längst auf strategische Schlüsselziele verständigt hat, führen die deutschen Gewerkschaften - obgleich ihre Tarifpolitik mehrheitlich nach wie vor gesamtwirtschaftlich begründet und konzipiert ist - unabhängig voneinander sektorale Tarifrunden. Es ist höchste Zeit, intensiv zu beraten, wie daraus so etwas wie eine gemeinsame Tarifbewegung entstehen kann.

Und in Europa? Hier sind die Gewerkschaften bei der Koordinierung der nationalen Tarifpolitiken in der letzten Dekade wenn auch nicht weit genug, so doch ein Stück vorangekommen. Dazu gehören Absprachen, sich bei der Lohnpolitik zumindest an den gesamtwirtschaftlichen Zielgrößen Preissteigerung und Produktivität $\mathrm{zu}$ orientieren, um lohn- und verteilungspolitisches Dumping auszuschließen. In den kommenden Jahren wird es darum gehen, dieser Zielstellung durch erfolgreiche

10 Haipeter, Th. (2003): Erosion der Arbeitsregulierung? Neue Steuerungsformen der Produktion und ihre Auswirkung auf die Regulierung von Arbeitszeit und Leistung, in: Kölner Zeitschrift für Soziologie und Sozialpsychologie 3, S. 521-542.

11 Vgl. ausführlich Ehlscheid, Ch. (2006): Entgrenzung von Arbeitszeit und Leistung - eine Herausforderung für die gewerkschaftliche Strategiebildung, in: Baukrowitz, A. et al. (Hrsg.) (2006): Informatisierung der Arbeit - Gesellschaft im Umbruch, Berlin, S. 223-235. Fergen, A. (2007): Schlechte Zeiten - Gute Zeiten. Mit gewerkschaftlicher Arbeitszeitpolitik zu "guter Arbeit", in: Peter, G. (Hrsg.): Grenzkonflikte der Arbeit. Die Herausbildung einer neuen europäischen Arbeitspolitik, Hamburg, S. 95-116.

12 Pickshaus, K. (2007): Gute Arbeit - vom Projekt zum gewerkschaftlichen Handlungsfeld. Versuch einer Zwischenbilanz, in: Peter, G. (2007), a.a. O. S. 341-356.

13 Vgl. zur Entwicklung der Einkommensverteilung und zur zunehmenden Lohnspreizung in Deutschland: DIW Berlin (2007), Wochenbericht 6, S. 7379. 
Lohnabschlüsse in der Praxis näher zu kommen. Darüber hinaus wird sich die europäische Koordinierung in zweifacher Hinsicht weiterentwickeln müssen: Zum einen gilt es, dem Druck der Standortkonkurrenz ein koordiniertes Vorgehen von Gewerkschaften und Betriebsräten auf Unternehmensebene entgegenzusetzen, um ein Ausspielen gegeneinander zu verhindern. Nur so kann es gelingen, auf Dauer lohnpolitische Erfolge in kollektiven Tarifverhandlungen oder Arbeitszeitstandards zu sichern. Zum anderen wird es darum gehen müssen, mittelfristig europaweit gemeinsame Forderungen im Bereich tariflicher Rahmenregelungen zu erheben und damit die Koordinierung auch auf qualitative Themen auszudehnen. So haben die im Europäischen Metallgewerkschaftsbund (EMB) zusammengeschlossenen Gewerkschaften bereits 2005 eine entsprechende Vereinbarung getroffen. Die ersten praktischen Erfahrungen sind beispielsweise mit der tariflichen Regelung von Weiterbildung gemacht und müssen bei der tarif- politischen Konferenz des EMB 2009 ausgewertet werden.

\section{MEHR VITALITÄT?}

Zurück zur eingangs formulierten Frage: Sind in der Tarifrunde 2007 in der Metallund Elektroindustrie Elemente der Revitalisierung der IG Metall sichtbar geworden oder hat diese Verteilungsrunde einen Beitrag dazu leisten können? Eine erste Antwort fällt positiv aus. Das verteilungspolitische Volumen des Abschlusses lässt auf eine merkliche Erneuerung der Verhandlungs- und Verteilungsmacht der IG Metall schließen. Und da das Ergebnis in weiten Bereichen auf hohe Akzeptanz der Mitglieder stößt und zum Teil die Erwartungen der gewerkschaftlichen Basis deutlich übertrifft, dürfte der Abschluss ebenfalls zu einer Stabilisierung der Mitgliederbindung geführt haben. Zudem sprechen aktuelle Zahlen im Bereich der Mitgliederentwicklung dafür, dass infolge der Tarifrunde ein deutlicher Anstieg der Neuaufnahmen ver- zeichnet werden kann. Dies alles lässt auf eine merkliche Konsolidierung der Organisationsmacht und eine Revitalisierung der IG Metall in der verhandlungspolitischen Dimension schließen.

Der zweite Teil der Antwort kommt nicht umhin, auf die noch zu leistenden Anstrengungen zu verweisen. Das gilt vor allem mit Blick auf die virulenten Strategiefragen, die im arbeitspolitischen Feld von der Neujustierung der Betriebs- und Tarifpolitik über die Rekonstruktion einer solidarischen Verteilungspolitik bis hin zur erfolgreichen Bearbeitung neuer, qualitativer Elemente der Tarifpolitik reichen. Die Tarifrunde 2007 stellte - grosso modo - einen wichtigen, ermutigenden Schritt in die richtige Richtung dar, doch der Weg aus der gewerkschaftlichen Defensive ist noch lang. Mit anderen Worten: Auch die IG Metall steht immer noch vor wichtigen $\mathrm{Zu}$ kunftsfragen, deren erfolgreiche Beantwortung unverzichtbar ist, soll dem Prozess der Revitalisierung Tempo und Nachhaltigkeit verliehen werden.

\section{Umsetzungsprobleme qualitativer Tarifverträge

Zunehmend enthalten Tarifverträge qualitative Inhalte, deren Umsetzung zugleich neue betriebspolitische Strategien der Tarifparteien erfordert. Ein Beispiel sind die Tarifverträge zur Qualifizierung (QualiTV), die die IG Metall zunächst 2001 in Baden-Württemberg und dann 2006 bundesweit abschließen konnte. Viele der darin erreichten qualitativen Regelungen werden für die Beschäftigten erst wirksam, wenn sie durch betriebliche Vereinbarungen und Abläufe konkretisiert werden. Aus den Erfahrungen in Baden-Württemberg werden im Folgenden Thesen zur Umsetzung qualitativer Tarifverträge abgeleitet.

Der „Tarifvertrag zur Qualifizierung in der Metall- und Elektroindustrie in BadenWürttemberg" ist mehr als eine Konkretisierung der bestehenden betriebsverfassungsrechtlichen Mitbestimmungsrechte zur betrieblichen Weiterbildung. Er geht auch weiter als der Lohn- und Gehaltsrahmentarifvertrag I für die Metallindustrie Nordwürttemberg/Nordbaden, in dem schon 1988 erste Regelungen zur betrieblichen Weiterbildung vereinbart, aber kaum im beabsichtigten Maße umgesetzt wurden. ${ }^{1}$

Die Regelungen in Kürze: Die Beschäftigten haben Anspruch auf ein jährliches Gespräch mit dem Arbeitgeber, in dem gemeinsam der Qualifizierungsbedarf festgestellt und ggf. Qualifizierungsmaßnahmen vereinbart werden. Kommt es zwischen Beschäftigten und Vorgesetzten zu keiner Einigung, so wird eine innerbetriebliche paritätische Kommission gebildet, zu der notfalls ein Vertreter der von den Tarifparteien gemeinsam getragenen „Agentur zur Förderung der betrieblichen Weiterbildung" (Agentur Q) mit Stimmrecht hinzugezogen wird. ${ }^{2}$ Parallel zum individuellen Anspruch auf Einigung im Qualifizierungsgespräch ist auch tariflich geregelt, wie der Betriebsrat in Planung und Umsetzung der Weiterbildung einzubeziehen ist.

Dabei wird ein umfassender Begriff betrieblicher Weiterbildung zugrunde gelegt, der nicht nur die Anpassung an veränderte Arbeitsaufgaben, sondern auch den Nachvollzug der ,ständigen Fortentwicklung des

\footnotetext{
Bahnmüller, R./Fischbach, S. (2006): Qualifizierung und Tarifvertrag. Befunde aus der Metallindustrie Baden-Württembergs, Hamburg, S.40 ff.

2 Weitere Aufgaben der "Agentur Q" sind z. B. die Entwicklung von Weiterbildungsmaßnahmen und die Beratung und Verbesserung der Informationen über Qualifizierungsangebote; Näheres dazu unter http://www.agentur-q.de.
}

Rainer Salm, IG Metall Bezirksleitung Baden-Württemberg. Arbeitsschwerpunkte: Unterstützung von Betriebsratsarbeit und Betriebsräte-Netzwerken, Arbeitspolitik/ Gruppenarbeit. e-mail: rainer.salm@igmetall.de Sylvia Stieler, Sozialwissenschaftlerin, IMU Institut Stuttgart. Arbeitsschwerpunkte: Arbeitsorientierte Forschung und Betriebsräte-Beratung. e-mail:sstieler@imu-institut.de 
fachlichen, methodischen und sozialen Wissens im Rahmen des eigenen Aufgabengebiets" (Erhaltungsqualifizierung) und eine Erweiterungsqualifizierung für „andere gleichwertige oder höherwertige Arbeitsaufgaben" umfasst. Außerdem haben Beschäftigte durch den Tarifvertrag einen Anspruch auf Wiedereinstellung, wenn sie bis zu drei Jahren für persönliche Weiterbildung ausscheiden.

Auch wenn einzelne Punkte der 2006 bundesweit vereinbarten Regelungen für die Metallindustrie von denen in BadenWürttemberg abweichen, stellt die gegenwärtige bundesweite Umsetzung Betriebe, Betriebsräte und Gewerkschaften vor vergleichbare Herausforderungen wie fünf Jahre zuvor in Baden-Württemberg.

\section{DER „QUALI-TV“ - ERFOLGSSTORY UND LERNFELD}

Die wissenschaftliche Evaluation der betrieblichen Umsetzung des „Quali-TV“ in Baden-Württemberg hat beeindruckend gute Ergebnisse erbracht. Die Zahl abgeschlossener Betriebsvereinbarungen stieg deutlich an, der Tarifvertrag bewirkte in einem Großteil der Betriebe nachweisbare Veränderungen der betrieblichen Weiterbildungspraxis und er wurde von den Betriebsräten sowie den Personalverantwortlichen mehrheitlich positiv bewertet. ${ }^{3}$ Gleichzeitig zeigte sich jedoch auch eine Reihe spezifischer Probleme, die typischerweise ein „automatisches “Wirksamwerden qualitativer Tarifverträge behindern und neue Handlungsansätze von den Interessensvertretungen erfordern. Dies wird anhand von sechs Thesen dargestellt. Sie beruhen auf Erfahrungen, die insbesondere im Rahmen eines von der IG Metall-Bezirksleitung initiierten Betriebsrätenetzwerkes mit ca. 20 Maschinenbaubetrieben über einen Zeitraum von drei Jahren gemacht wurden. ${ }^{4}$

\section{GUT DING WILL WEILE HABEN}

Die Umsetzung des Tarifvertrages in den Betrieben hat sich als ein langwieriger Prozess herausgestellt, der mehr Zeit in Anspruch genommen hat, als alle Beteiligten erwartet hatten. Es geht dabei nicht um Monate, sondern um Zeiträume von zwei bis drei Jahren. Der Grund dafür lag nicht so sehr in besonders gravierenden Interessenkonflikten, sondern in der Schwierigkeit, die komplexen innerbetrieblichen
Veränderungen tatsächlich in Gang zu bringen, die mit der Vorbereitung und Auswertung der jährlichen Qualifizierungsgespräche und der Umsetzung des häufig erstmals sichtbar werdenden Qualifizierungsbedarfs zusammenhängen (bis hin zur Neugestaltung entsprechender EDVSysteme etc.). Gleichzeitig standen sowohl Management als auch Betriebsräte ständig unter dem Druck, anderen Themen (wie Standortsicherung, ERA-Einführung oder laufende Tarifrunden) Vorrang einzuräumen. Die Zeitdauer des Umsetzungsprozesses ist eine Herausforderung sowohl für bisherige gewerkschaftliche Betreuungsund Qualifizierungsformen als auch für die Arbeitsweise von Betriebsräten. Gewerkschaften müssen zusätzlich zur einmaligen Schulung über Tarifvertragsinhalte eine mehrjährige Prozessbegleitung in Gang bringen. Betriebsräte müssen zusätzlich zum Abschluss einer Betriebsvereinbarung z. B. die Qualifizierungsgespräche ständig beratend begleiten und in Gang halten. Insbesondere für Klein- und Mittelbetriebe ist angesichts der Ausdünnung von Vorgesetzten- und Overhead-Strukturen eine systematische Weiterbildung eine sichtbar schwierige Aufgabe. In Baden-Württemberg ist es über Projekte wie das Betriebsrätenetz Maschinenbau gelungen, längerfristige wirksame Unterstützungsstrukturen auszubauen. Ein flächendeckendes Angebot konnte damit jedoch nicht bereitgestellt werden.

\section{ALLER ANFANG IST SCHWER}

Gerade bei der ersten Umsetzung des Tarifvertrags ist häufig ein Bruch zwischen der Bedarfsermittlung und der Umsetzung der für nötig befundenen Maßnahmen zu beobachten. Die tarifvertraglich vorgegebene jährliche Durchführung von Qualifizierungsgesprächen ist auch für die Arbeitgeberseite sachlich meist einleuchtend. Andererseits stehen Betriebe vor der erheblichen Herausforderung, innerhalb eines Jahres Gespräche mit allen Beschäftigten (für manche Vorgesetzte sind es 30 bis 40) durchzuführen, auszuwerten und daraus in Abstimmung mit dem Betriebsrat Qualifizierungsmaßnahmen abzuleiten, zu planen, zu realisieren und im Idealfall vor der nächsten Runde eine eigene Auswertung des betrieblichen Qualifizierungsgeschehens vorzunehmen. In vielen baden-württembergischen Betrieben zeigte sich, dass die jährlich neue Gesprächsrunde auf einen
„Maßnahmestau“ aus dem Vorjahr trifft. Mit zunehmender Routine und Einsatz entsprechender finanzieller Mittel lässt sich dieser verringern, gleichwohl baut sich hierdurch Unmut auf und es besteht die Gefahr, dass die Qualifizierungsgespräche entwertet werden.

\section{VERBINDUNG VON INDIVIDUELLEN UND KOLLEKTIVEN RECHTEN}

Die Ermittlung des Qualifizierungsbedarfs - als Grundlage einer strategischen Personalentwicklung - erfolgt einerseits „topdown“, im Idealfall gemeinsam durch Geschäftsleitung und Betriebsrat, ausgerichtet an der künftigen Unternehmens- und Produktstrategie. Mit dem tariflichen Anspruch auf Qualifizierungsgespräche ${ }^{5}$ wurde nun eine gegenläufige, „bottom-up“orientierte Form der Bedarfsermittlung eingeführt. Damit können persönliche Weiterbildungsinteressen besser eingebracht und betriebliche Planungs- und Entscheidungsabläufe partizipativer gestaltet werden. Diese zunächst voneinander unabhängigen Verfahren der Bedarfsermittlung müssen jetzt miteinander verschränkt werden. Im Idealfall sollten sich die Beschäftigten mit Kenntnis der Unternehmensstrategie auf ihre Qualifizierungsgespräche vorbereiten und den eigenen Bedarf formulieren. Die mit dem Tarifvertrag beabsichtigte Erhöhung der Partizipationschancen der Beschäftigten erfordert jedoch eine Unterstützung für einzelne Mitglieder, die nicht nur ihre Ansprüche aus dem Tarifvertrag kennen, sondern sie auch individuell einfordern müssen.

\section{ZWISCHEN KONFLIKT UND KOOPERATION}

Bei den betrieblichen Umsetzungsverhandlungen war es für viele Betriebsräte schwierig, die richtige Mischung aus Konflikt und Kooperation zu finden. Viele Arbeitgeber hatten zwar ein Interesse an einer vereinbarten Systematisierung innerbe-

\footnotetext{
3 Bahnmüller, R./Fischbach, S. a.a.O., S. 198 ff. (vgl Fn. 1)

4 IG Metall BaWü (2005): Starke Betriebsräte in einem starken Netz. 3 Jahre Betriebsräte-Netzwerk Baden-Württemberg; CD-ROM, Stuttgart.

5 Die Gespräche können als Einzel- wie als Gruppengespräche geführt werden. Einzelheiten sollten aus gewerkschaftlicher Sicht in Betriebsvereinbarungen geregelt werden.
} 
trieblicher Weiterbildungsabläufe. Sie wollten aber keine Ausweitung individueller Ansprüche und keine Ausweitung der Mitbestimmungspraxis zulassen, da sie eine schwer abschätzbare Ausweitung der Weiterbildungskosten fürchteten. Sollte der Fortschritt des Tarifvertrages nicht nachträglich wieder verloren gehen, mussten die Betriebsräte in harten Konflikten um die Realisierung dieser Kernbestandteile ringen. Andererseits zeigen alle Erfahrungen, dass neue Weiterbildungsstrukturen von Beschäftigten und Vorgesetzten nur dann angenommen werden, wenn sie von Geschäftsleitung und Betriebsrat gemeinsam getragen werden. Trotz der Interessenkonflikte mussten also in den Verhandlungen auch Kooperationsgrundlagen für eine gemeinsam getragene Umsetzung geschaffen werden. Damit Betriebsräte bei solch widersprüchlichen Anforderungen weder resignieren noch sich in Phyrrussiegen verzetteln, ist ein lebendiger Erfahrungsaustausch zwischen allen erforderlich, die sich auf diesen schwierigen Weg gemacht haben. Dies kann mehr bewirken (und ist besser zu realisieren) als eine prozessbegleitende Einzelberatung für jeden Betriebsrat.

\section{VIELE WEGE FÜHREN NACH ROM}

Die Startbedingungen für die Umsetzung des Tarifvertrags waren in den Betrieben äußerst unterschiedlich. Während einige bereits eine gewisse Tradition der betrieblichen Weiterbildung hatten, jährliche Weiterbildungsprogramme veröffentlichten und in breiterem Umfang Maßnahmen anboten, lief in anderen Betrieben außer Managementschulungen überhaupt nichts. Entsprechend unterschiedlich mussten die Ziele bestimmt werden, die erreicht werden sollten. Während einige Betriebsräte schon die Ernennung eines Weiterbildungsverantwortlichen oder die Einführung von Teilnahmebescheinigungen als
Anfangserfolg verbuchten, konnten andere erst zufrieden sein, wenn bisher benachteiligte Beschäftigtengruppen am bereits bestehenden Weiterbildungsangebot partizipieren konnten. Die traditionelle Vorgabe einer Musterbetriebsvereinbarung als gewerkschaftliche Standardvorgehensweise kam deshalb schnell an ihre Grenzen. In etlichen Betrieben funktionierten solche Vereinbarungen, in anderen allerdings nicht. Trotz abgeschlossener Betriebsvereinbarung veränderte sich dort an den betrieblichen Abläufen wenig. In anderen Betrieben wiederum, in denen anfänglich der Abschluss einer Betriebsvereinbarung aus Sicht der Betriebsräte kaum durchsetzbar schien, machte man sich zunächst ohne sie auf den Weg und konnte allmähliche, aber doch beachtliche Verbesserungen betrieblicher Abläufe erreichen. Die Vorgehensweisen waren und sind bis heute unterschiedlich. Gefordert waren die Organisation eines gemeinsamen Lern- und Suchprozesses und eine „Leitplankendiskussion“, wie sie zum Themenstrang „Umsetzung des Qualifizierungstarifvertrags“ im „BetriebsräteNetzwerk Baden-Württemberg "6 angeboten wurde.

\section{MITGLIEDERVORTEILE NUR DURCH ZUSÄTZLICHE BERATUNG}

Eine qualifizierte Umsetzung des Tarifvertrags verändert in vielen Fällen die betrieblichen Abläufe und führt zu Verbesserungen der Einflusschancen der Beschäftigten, von denen nicht nur die Gewerkschaftsmitglieder profitieren. Um deren Nutzen zu optimieren, müssten - ergänzend zur Tätigkeit des Betriebsrats - eine eigenständige Ansprache der Mitglieder durch gewerkschaftliche Akteure stattfinden und Materialien bereitgestellt werden, die dessen Position bei der Durchsetzung individueller Ansprüche stärkt (Ansätze in diese Richtung sind der Job-Navigator ${ }^{7}$ und die Checklisten zur Analyse der eigenen Kom- petenz („KORA“) und der Arbeits- und Lernsituation („SALSA“)). ${ }^{8}$

Gerade mit der großen Bedeutung der Vertretung individueller Interessen im Qualifizierungsgespräch bietet der Tarifvertrag zur Qualifizierung hier einen idealen Ansatzpunkt, erfordert aber gleichzeitig (trotz absehbar knapper Personalressourcen) die Ergänzung der Mitgliederbetreuung um Beratungsangebote.

\section{QUALITATIVE TARIFPOLITIK, AKTIVIERENDE BETRIEBSPOLITIK}

Mit der tariflichen Regulierung qualitativer Themen wie Qualifizierung oder Innovation unterstreichen die Gewerkschaften ihren Anspruch, dass sie sich um eine „Arbeit mit Zukunft" ebenso kümmern wie um die Erhöhung der Kaufkraft. ${ }^{9}$ Mit dem Abschluss solcher Tarifverträge ist es für die Gewerkschaften aber nicht getan. Viele qualitative Regelungen werden für die Beschäftigten erst wirksam, wenn die betriebliche Umsetzung langfristig begleitet wird. Dies erfordert neuartige Unterstützungsformen und eine aktivierende Betriebspolitik der Gewerkschaften. Die Umsetzung von Qualifizierungstarifverträgen kann und sollte hierfür zum Versuchsfeld gemacht werden.

6 Bahnmüller, R./Fischbach, S. (2004).: Evaluationsbericht zum Themenstrang "Umsetzung des Qualifizierungstarifvertrags" im Projekt „Betriebsräte-Netzwerk Baden-Württemberg " der IG Metall (http://www2.igmetall.de/homepages/brnetzwerk-ba-wue/file_uploads/evaluationsberichtbahnmueller_fischbach.pdf)

7 Zum Job Navigator vgl. http://www.igmetall.de/ cps/rde/xchg/SID-0A342C90-608104A3/internet/style.xsl/view 3598.htm

8 Vgl. zu den Kompass-Checklisten: http://www. agenturq. de/service/thema.html?id=10

9 Burkhard, O./Iwer, F./Wagner, H. (2006): Tarifrunde 2006 - Perspektive inklusive - Bewertung und Ausblick, in: WSI-Mitteilungen 9, S. 513-516. 